\title{
KAISER LEON III ALS OZIAS, KÖNIG DER JUDÄER?
}

\author{
Hiroshi WADA*
}

Heute sind sich viele Historiker des Mittelalters, aber erst recht die Byzantinisten dessen bewußt, daß es ihnen kaum gelingen würde, die Motive des ersten ikonoklastischen Kaisers, Leon III. (717-741), über den Verbot des Ikonenkultes befriedigend zu interpretieren.(1) Denn die diesbezüglichen Quellen sind nach dem Sieg des Bilderverehrers im 7. Konzil zu Nikaia (24. Sept.-23. Okt. 787) aus erklärlichen Gründen so gründlich vernichtet worden, daß man sich auch an diesem Punkt mit Vermutungen zufrieden geben muß.(2)

Die gängige Erklärung in der einschlägigen Literatur will in ihren Grundzügen die Motive im Zusammenschluß zweier Elemente suchen. 1. Die latente Bilderfeindlichkeit in den östlichen Provinzen des Reichs. Von dort stammen alle ikonoklastischen Kaiser, einschließlich des Kaisers Leons III. Daher sind sie fortwährend durch die bilder-feindlichen Neigungen seitens der Juden, der Paulikianer und der Araber vor allem beeinflußt worden. 2. Das ikonoklastische Edikt des Kalifen Jezids II. (720-724 n. Chr.). Dieses Edikt gab dem Kaiser Leon III. den direkten Anlaß, auch in seinem Lande den Bilderkult zu verbieten. ${ }^{(3)}$

Bekanntlich hinterließ Theophanes Confessor in seiner Chronographia, unserer Hauptquelle, vier Stellen, die sich auf die Anfänge des Bilderstreites beziehen: a.m. 6215 (I.), a.m. 6217 (II.), a. m. 6218 (III.) und a.m. 6221 (IV.). Von diesen vier Berichten hat nur Nr. IV. einen Parallelbericht bei Nikephoros in seinem sog. Breviarium.

Wir beginnen mit dem inhaltlich gesicherten Bericht, d. h. mit der Nummer IV. Dieser Bericht wird von den Forschern einstimmig als der offizielle Anfang des byzantinischen Bilderstreites anerkannt. Dort heißt es: „Am 7. Tag des Monats Januar der 13. Indiktio, am 3. Tag der Woche berief der gottlose Leon eine Ratssitzung gegen die heiligen und verehrten Bilder im Tribunal der 19 Beisitzer. Er ließ den heiligsten Patriarchen Germanos zu sich kommen, in der Überzeugung, daß er die Anordnung gegen die heiligen Bilder unterschreiben

* Prof. an der Tôyô Eiwa Jogakuin Hochschule, Tôkyô. 
würde. Aber dieser hochwürdige Knecht des Christus wurde gar nicht durch seine abscheuliche Lehre überredet.“(4) Anno mundi 6221 bedeutet in der Tat a. m. 6222, d. h. das Jahr 730 n. Chr., weil hier bekanntlich Theophanes bei der Jahresberechnung ein Fehler unterlaufen ist. ${ }^{(5)} \mathrm{Zu}$ diesem Zeitpunkt steht der Entschluß des Kaisers schon fest, daß er den Bilderverbot offiziell kundgeben wollte. Nach der Weigerung des amtierenden Patriarchen Germanos, der sich gleich danach zurückzog, nominierte er seinen Gesinnungsgenossen Anastasios als den neuen Patriarchen und ließ den Bilderverbot öffentlich bekanntgeben.

Allerdings wissen wir, daß er sich drei Jahre davor schon ikonoklastisch eingestellt war. Nämlich a. m. 6218 berichtet Theophanes folgendes Ereignis:

„,Die Bewohner in der kaiserlichen Stadt waren mit seiner neuen Lehre sehr unzufrieden und besorgt. Sie griffen einige kaiserliche Beamten an und töteten sie, weil sie die Ikone des Herrn Jesu Christi am Tor der großen Chalke niederrießen. "(6) Daß diese Anordnung, die seit der Zeit des Kaisers Maurikios (582-602 n. Chr.) bekannte Ikone am Palasttor verschwinden zu lassen, vom Leon III. erteilt wurde, steht fest. Denn ein Jahr zuvor, d.h. 726 n. Ghr., zeigte er sich schon öffentlich, sich gegen den Ikonenkult zu stellen. (II.)

Dort heißt es: „In diesem Jahr (=a. m. 6217) begann der gottlose Kaiser Leon zu behaupten, die heiligen und verehrten Bilder zu stören. Als Gregorius, der Papst von Rom, davon erfuhr, ....' schrieb er an Leon einen dogmatischen Brief, daß ein Kaiser über den Glauben kein Wort aussprechen solle und daß er das altehrwürdige Dogma der Kirche, das von den heiligen Vätern gelehrte Dogma nicht ändern solle."(7) Diese Stelle zeigt, daß seine Absicht, den Ikonenkult in Abrede zu stellen, so weit bekannt war, daß sie sogar vom fernen Rom eine negative Reaktion erhalten hatte.

Vom Zeitpunkt der öffentlichen Bekanntgabe im Jahre 730 bis zum Zeitpunkt seiner persönlichen Äußerung im Jahre 726 sind vier Jahre verflossen. Aber daß er nicht erst mit dem Jahr 726 angefangen hat, den Ikonenkult zu verbieten, zeigt noch eine Stelle bei Theophanes a. m. 6215 (=724 n. Chr.) (I.).

Dort heißt es: ,In diesem Jahr erschien ein gewisser Jude aus Laodikeia am küstengelegenen Phoinike. Er war Zauberer. Er kam zu Jezid (II.) und teilte ihm mit, er würde 40 Jahre die Herrschaft über die Araber innehaben, wenn er die in den Kirchen der Christen in seinem Reich verehrten ehrwürdigen Ikonen vertilgen würde. Davon überzeugt der ungebildete Jezid, beschloß die allgemeingültige Vorschrift gegen die heiligen Bilder. Aber durch Gnade 
unseres Herrn Jesu Christi und durch Unterstützung seiner unverdorbenen Mutter und aller Heiligen starb in diesem Jahr Jezid, so daß viele Leute keine Kunde von seiner teuflischen Vorschrift erhalten hatten. Leon, der Kaiser, wurde dieser verhaßten und gottlosen Häresie teilhaftig und wurde Ursache vieler Ubel. Er fand als seinen Gesinnungsgenossen dieser Ungebildetheit einen gewissen Beser mit Namen. Dieser war Kriegsgefangene aus der Reihe der Christen in Syrien und wurde vom Glauben in Jesus Christus abtrünnig und wählte sich die Dogmen der Araber. Dieser wurde vor nicht zu langer Zeit von jener Knechtschaft befreit und wurde Bürger des Römerreichs. Er wurde wegen seiner Körperkraft und der gleichen Gesinnung der Häresie von diesem Kaiser hoch geschätzt. Dieser wurde für den Kaiser der Kampfgenosse dieses großen Unheils.“( $(8)$

Die wichtigste Aussage in dem obigen Zitat liegt für unsere Eragestellung unzweifelhaft in dem Punkt, daß Kaiser Leon III. vom Edikt des Kalifen nicht nur Kunde erhalten hatte, sondern wohl ihm seine Zustimmung gegeben und gar einen Syrer namens Beser als seinen Helfershelfer gefunden hatte. Dieser hatte wohl später deswegen die Würde eines Patriziers erhalten (siehe unten). Es ist bekannt, daß die Forscher über die Existenz dieses Syrers im Zweifel sind.(9) Aber wir dürfen ihn wohl nicht einfach als eine legendäre Person abtun. Denn von diesem gleichen Beser spricht Theophanes noch zweimal:

A. m. 6218 heißt es: ,daß er (=Beser) ein gottverneinender Kampfgenosse vom Keiser Leon III. ist und daß er mit ihm in der gleichen Unvernunft wetteifert. Die beiden Männer waren voll von Ungebildetheit, wodurch viele Ubel entstanden.“(10) Ferner heißt es von ihm a. m. 6233: „,daß er, Patrizier Beser, dieser Freund der Sarazener, vom Usurpator Artabasdos .... mit großen Schmerzen getötet wurde." "(11)

Die Aussage von Theophanes vom Jahre 724 n. Chr. dürfen wir in ihren Grundzügen als echt gelten lassen, da wir einen ganz ähnlichen Bericht von seinem Zeitgenossen, dem Presbyter Johannes von Jerusalem haben.(12) Allerdings stehen bei ihm folgende 3 Punkte anders als bei Theophanes: 1. Der Name der Person, die dem Kalifen 40 jährige Herrschaft versprach, hieß nicht ein ungenannter Jude aus Laodikeia, sondern Tessarakontapechus, ein ,vierzig Ellen hoher," jüdischer Magier aus Tiberias.(18) 2. Jezid II. wurde von ihm eine 30 jährige Herrschaft zugesprochen, statt eine 40 jährige. 3. Jezid II. starb etwa zwei und halb Jahre später als nach der Veröffentlichung des Ediktes (d. h. im Jahre 721 n. Chr.). Nach Theophanes aber im Jahre 724 n. Chr.(14) 
Diese drei Unterschiede sollen aber nicht zu schwer in die Waagschale geworfen werden. Geschweige denn sie deswegen als legendär verwerfen. Eher soll den gemeinsamen Grundrissen der Erzählungen sowohl bei Theophanes als auch bei Johannes unsere gesteigerte Aufmerksamkeit gewidmet werden. Denn dadurch können wir uns etwa den chronologischen Ablauf der Anfänge des Bilderstreites vorstellen: 1. Kaiser Leon III. erfuhr vom Edikt des Kalifen im Jahre 721 bzw. 724 n. Chr. und fand schon in diesem Stadium seinen Helfershelfer in einem Syrer namens Beser. 2. Dann im Jahre 726 n. Ghr. begann er sich gegen die Bilder einzustellen. 3. Und er ließ 727 n. Chr. die bekannte Ikone des Herrn Jesu Christi am Tor der Chalke des Palastes entfernen. 4. 730 n. Chr. veröffentlichte er das Edikt gegen den Bilderkult. Bemerkenswert scheint es uns, daß Kaiser Leon III. schon im ersten Stadium, als er vom Edikt des Kalifen erfuhr, offenbar seine Zustimmung sofort gab. Warum er dann dazu geneigt war, lautet die gängige Antwort, hier auch dem Theophanes, Chronographia a.m. 6218 folgend, weil ,dieser Leon gleiche Gesinnung wie

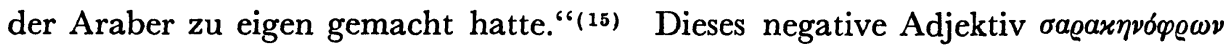
wurde auch dem genannten Beser beigefügt und wurde von Theophanes in der Verbindung der Araber mit den Ikonoklasten gebraucht. Nach der Beschreibung von Theophanes wurde Leon III. also unter dem Einfluß der islamischen Bilderfeindlichkeit zu Ikonoklasten geworden.

$\mathrm{Da}$ die Antwort von Theophanes zu bescheiden lautet und uns nicht zufrieden stellt, wollen wir uns noch weiter fragen, was der Kaiser für eine innere Voraussetzung gehabt hatte, um solche Ansicht zu eigen zu machen. Denn ihm müßte es ja klar gewesen sein, daß er sich dann mit seinem Vorhaben gegen die jahrhundetlange Tradition des offiziellen Glaubens in der orthodoxen Kirche, den er als Kaiser dieses Reiches offiziell zu vertreten hatte, stemmte.

Unsere nächste Quelle ist ein Brief vom Papst Gregorius II. an den Kaiser Leon III. Er ist jetzt neben einer kürzeren Redaktion nur in der griechischen Übersetzung erhalten und dürfte wohl mit dem 2. Bericht von Theophanes im Zusammenhang stehen, ohne daß wir ihn allerdings genau datieren können. ${ }^{(16)}$

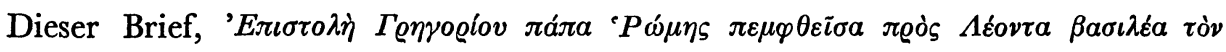

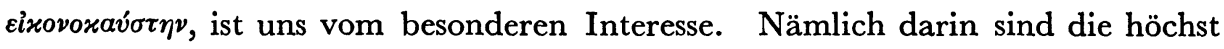
persönlichen Ansichten dieses Kaisers über den Ikonenkult vermerkt. Im folgenden seien drei typische Aussagen zitiert, die für seine Auffassungen charakteristisch sind. 1. In den Zeilen 37-39 heißt es in der Antwort von Gre- 
gorius II. an Leon III.: „Du (=Leon III.) schriebst, daß es nicht nötig sei, die von den menschlichen Händen gemachten Ikonen und die sämtlichen Gestalten gemäß dem Urbild, wie Gott sagte, zu verehren, weder im Himmel noch auf Erden.“(17) 2. In den Zeilen 156-157 heißt es: „Du (=Leon III.) behauptest, daß wir die Steine, die Wände und das Brettchen anbeten. Es stimmt aber nicht, wie Du behauptest, o Kaiser!“(18) Und schließlich in den Zeilen 179-183 heißt es: „Du (=Leon III.) schriebst, Ozias, der König der Judäer, habe nach 800 Jahren die eherne Schlange aus dem Tempel vertilgt. Ich habe nach 800 Jahren die Götzenbilder aus der Kirche vertilgt. Wirklich war Ozias Dein Bruder und hatte Deine Gesinnung und tyrannisierte die damaligen Priester wie Du.“(19) In diesen Zitaten kommen sehr deutlich die persönlichen Auffassungen vom Kaiser Leon III. zum Ausdruck, daß er in den Ikonen gar keine geistige Widerspiegelung des Göttlichen anerkennen will, weil es sich dabei nur um ,ein Brettchen" handelt, und daß er daher gar nicht für nötig hielt, sie anzubeten. Sogar klingt der Selbstvergleich mit dem vermeintlichen König der Judäer, so negativ, daß er die Ikonen und den Ikonenkult gleichsam als etwas Schädliches angesehen hatte, wie damals die eherne Schlange im Tempel. Das Schädliche müßte für seine realistischen Angen sicher in den extremen Ikonenkult und dem übermäßigen Wunderglauben an die Ikonen, wie etwa an die Acheiropoieta, gelegen haben. Denn je fester man daran glaubt, desto wirklichkeitsfremder und unrealistischer wird man. Gerade dieser Punkt, daß der übertriebene Ikonenkult die der nüchternen Wirklichkeit entspechenden Handlungen und Reaktionen zu sehr verhindert, müßte für seine bilderfeindliche Stellungnahme ausschlaggebend gewesen sein. Nicht primär aus den dogmatischen Überlegungen oder aus mönchsfeindlichen Einstellungen oder aber aus der ökonomischen Kalkür wurde er bilderfeindlich. Sondern von seiner praktischen Veranlagung her müßte er schon, bevor er vom bilderfeindlichen Edikt des Kalifen erfuhr, dazu abgeneigt gewesen sein, sich blindlings dem Ikonenkult zu bekennen. Der starke Wirklichkeitssinn dieses Soldatenkaisers wollte vor allen Dingen das bilderfreundliche Brauchtum seiner Zeit strikt ablehnen. ${ }^{(20)}$

$\mathrm{Ab}$ 6. Jahrhundert n. Chr. verbreitete sich bekanntlich der Ikonenkult immer breiter und tiefer im Lande der Byzantiner, als dessen Zenit die Legende von der Acheiropoieta anzusehen ist.(21) Man ehrte die Ikonen durch Kranzlicht, Weihrauch, Kuß, Waschung und Proskynese. Da jede Ikone als die pneumatische Wiedergabe des Gemalten gilt, glaubte man fest an die geheim- 
nisvollen Kräfte der Ikonen wie etwa an die Heilskräfte der wundertätigen Ikon von Sozopolis. Oder man glaubte an die sprechende Ikon wie etwa die heilige Ikon des Herrn Jesu Christi am Tor der Chalke des Palastes, die im Traum dem Kaiser Maurikios seinen Rat erteilt haben sollte. ${ }^{(2)}$ Die Ikonen wurden auch als Schutzheiligen sowohl der einzelnen Person als auch der ganzen Stadt angesehen und wurden als solche am Eingangstor der Stadt angebracht, wie etwa im Jahre 626 n. Chr. oder 1453 n. Chr., als diese kaiserliche Stadt von den Feinden schwer belagert wurde. Oder man trug sie mit in den Krieg, um dadurch die himmlischen Kampfgenossen zu bekommen. Oder man schwörte vor den Ikonen, man wählte die bestimmte Ikon zum Paten bei der Taufe eines Kindes. Und man baute eigens zu ihrer Verehrung die Kirche. Kurz, die Ikonen waren überall zu sehen und zu verehren, sowohl in Privathäusern als auch in öffentlichen Gebäuden, ja sogar auch in Gefängnissen. Und der Ikonenkult war im Alltagsleben der Byzantiner sowohl in der Hauptstadt als auch in den Provinzen so fest eingesessen, daß man nunmehr gar keine orthodoxe Kirche ohne Ikonen denken konnte.

Aber der an der Praxis orientierte Geist dieses kühnen Soldatenkaisers wollte solches Gedeihen des Ikonenkultes gerade nicht als wünschenswert begrüßen. Derselbe praktische Geist scheint auch in seiner Gesetzgebung, Ekloge, durchgeschlagen zu haben. ${ }^{(23)}$ Sowohl in der Auswahl der Stoffe als auch einige Stellen im Prooimion der Ekloge scheinen dies beizupflichten. Gesammelt wurden darin von der Komission all diejenige Fälle, die keine juristisch-theoretischen, sondern zweckdienliche und der Wirklichkeit passende Lösungen im Alltagsleben forderten: Verlobung und Ehe, Mitgift und Schenkung, Testament und Erbfolge, Waise und Vormundschaft, Freigelassene und Rückfall, Kaufvertrag, Darlehen, Hinterlegung, Pacht, Miete, Zeuge, Dialysis, Soldatengüter, Strafe und Verteilung der Kriegsbeute wurden in 13 Kapiteln zusammengefaßt.

Im Prooimion erklärte Leon III. seine Absicht folgendermaßen: ,damit die Urteile und die dem Verbrechen entsprechenden Strafen in diesem Buch klar und ausführlich passenderweise registriert werden.“(24) Ferner beschrieb er, daß ,diejenigen, die Verstand und Einsicht besitzen und die echte Gerechtigkeit gründlich kennen, können auf gerechter Weise Urteile fällen."(25) In der nichtrhetorischen, sondern in der schlichten Sprache des Textes, der zweckmäßigen Auswahl der Stoffe sowie der immer wiederkehrenden Hervorhebung des gesunden Menschenverstandes scheint die klare Absicht dieses realistischen 
Herrschers angedeutet zu sein.

Sein realistischer Charakterzug dürfte auch durch die damalige Situation des Reiches noch gestärkt worden sein. In den Zeiten, in denen Leon III. seine Karriere als Spatharios und enger Freund des Kaisers Justinianos II. begann und schließlich durch die Rebellion den Thron des byzantinischen Kaisers erlangte, erlebte er im Innern des Reiches die fortwährenden Aufstände und Unruhen, die unvermeidlich die Standhaftigkeit des Reiches nur schwächten. Als Zeitgenosse mußte er außenpolitisch den Verlust des nordafrikanischen Reichsgebiets, die Entstehung des Ersten Bulgarischen Reiches (681-971) im byzantinischen Territorium und den sichtbaren Abstieg des byzantinischen Machtbereichs vom Exarchat in Ravenna mitansehen. Und nicht zuletzt die seit 672 n. Chr. jährlich wiederholten Angriffe der Araber auf die Hauptstadt, deren bekannteste und schwerste in den Jahren 717-718 n. Chr. er selbst als Kaiser mit Hilfe des griechischen Feuers und der großen Naturkatastrophe zurückschlagen konnte, verschlimmerte nur die Lage.

Leon III. wurde und wird immer noch als siegreicher Verteidiger der orthodoxen Welt gegen die islamischen Eroberungswellen gerühmt. Auch die Veröffentlichung der Ekloge wurde und wird immer noch als seine positive Leistung angesehen. Im Gegensatz dazu wurde und wird immer noch sein Bilderverbot gleichsam als ein böser Makel seiner Herrschaft sehr negativ bewertet. Negative Bewertung wohl deswegen, weil die Forscher, meistens dem Theophanes folgend, seinen Bilderverbot allzusehr vom orthodoxen Standpunkt her zu begreifen pflegten. Dabei scheint die übliche Gegenüberstellung der orthodoxen Bilderverehrung mit der häretischen Bilderverneinung gewisse Rolle zu spielen. Aber wenn wir sein ganzes Unternehmen einschließlich des Bilderverbotes unvoreingenommen mustern, soweit dies quellenmäßig möglich ist, dann dürfte unser Urteil nicht verfehlt sein, daß er sein ganzes Unternehmen konsequent vom realistischen Standpunkt her geplant und in die Tat umgesetzt hat. Auch der Bilderverbot dürfte für ihn nicht anders als ein realistischer Eingriff in den religiösen Bereich gewesen sein. Falls unser Urteil zutreffen sollte, dann müßte das bisherige Bild dieses Kaisers als des ersten ikonoklastischen Kaisers im kritischen Sinne korrigiert werden. Dann müßte das negative Urteil über seinen Bilderverbot aufgehoben werden. ${ }^{(26)}$ 


\section{Anmerkungen}

(1) Von der älteren bis zur jüngeren Literatur ist diese Unzulänglichkeit festzustellen: K. Schwartzlose, Der Bilderstreit. Ein Kampf der griechischen Kirche um ihre Eigenart und um ihre Freiheit, Gotha 1890, K. Schenk, Kaiser Leons III. Walten im Innern, in: BZ. 5, 1896, 257-301, A. A. Vasiliev, History of the Byzantine Empire, I. Madison and Milwaukee 1964 (=1928), 251-259, G. Ostrogorsky, Geschichte des byzantinischen Staates, München 1964, 130-137, H. G. Beck, Die griechische Kirche im Zeitalter des Ikonoklasmus, in: Kirchengeschichte, hrsg. H. Jedin u. a., Bonn 1973, Bd. III, I., 2. Abschnitt, 31-61. seien hier stellvertretend genannt.

(2) Zur Liste der übrig gebliebenen Quellen siehe Ostrogorsky, a. a. O., 123-126, Beck, a. a. O., p. 31. Natürlich hat diese Lücke der Quellen dazu geführt, daß sie die Forscher mit verschiedenen Hypothesen zu erfüllen versucht haben. vgl. dazu Anm. 26.

( 3 ) vgl. Anm. 8 und 9.

(4) Theophanes, Chronographia, a. m. 6221 (de Boor, 408, 31-409, 14. Auswahl):

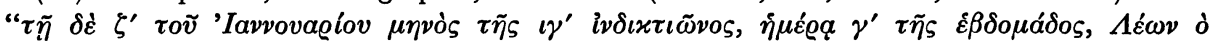

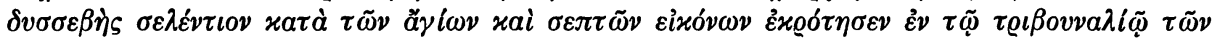

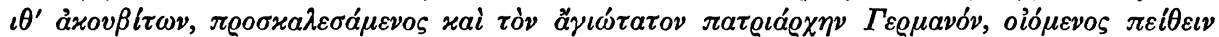

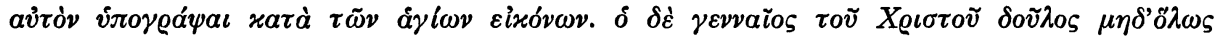

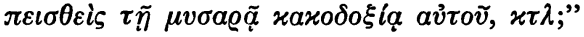

( 5 ) G. Ostrogorsky, ,Theophanes“, in: RE. 2. Reihe, V., 2127-2132, G. Moravcsik, Byzantinoturcica I. Berlin 1958, 531-537, F. Dölger, Das Kaiserjahr der Byzantiner, München 1949 (Sitzungsberichte der Bayerischen Akademie der Wissenschaften, Philos.-hist. Kl. 1949, Heft 1).

(6) Theophanes, Chronographia, a. m. 6218 (de Boor, 405, 5-405, 8.):

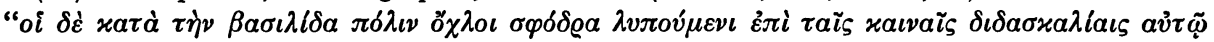

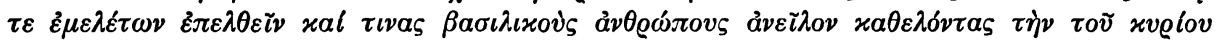

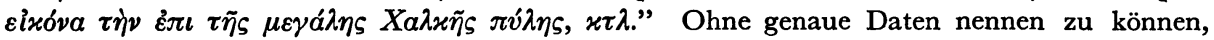
wissen wir, daß Leon III. versuchte, die Bewohner der Hauptstadt zu überreden. (Vita Stephani, in: MPG. 100, 1084 B. Nikephoros, Breviarium 57, 26).

(7) Theophanes, Chronographia, a. m. 6217 (de Boor, 404, 3-404, 9):

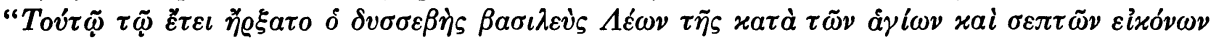

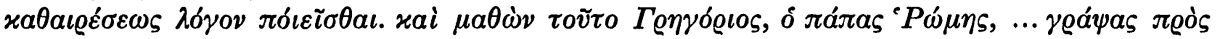

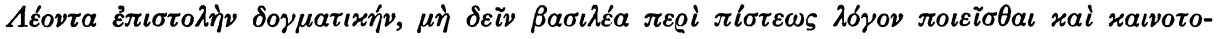

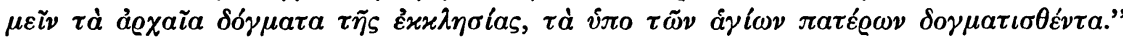

(8) Theophanes, Chronographia, a. m. 6215 (de Boor, 401, 29-402, 16):

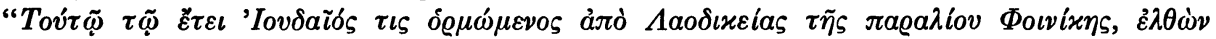

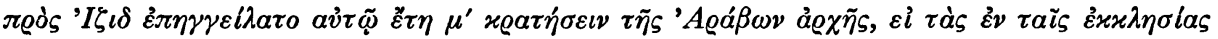

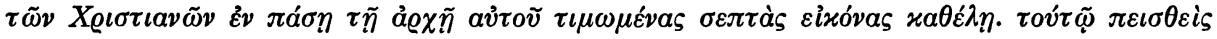

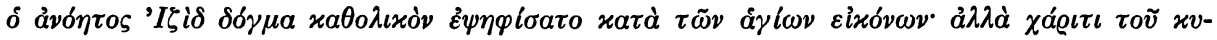

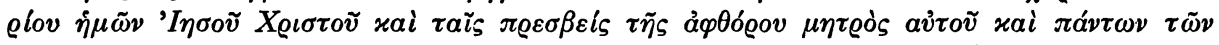

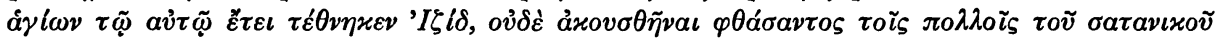

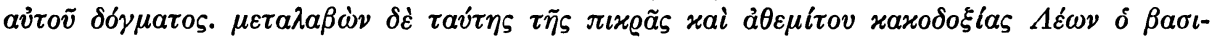

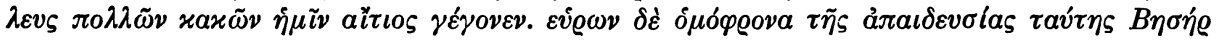

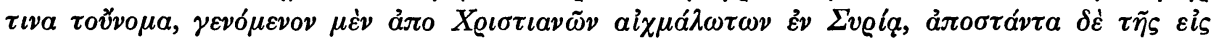

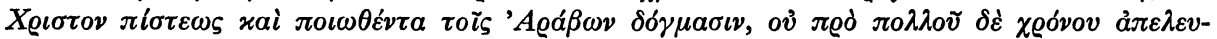

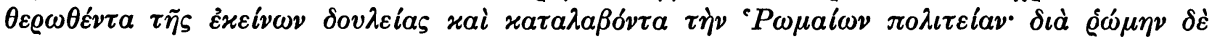

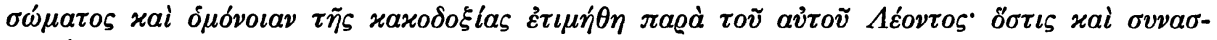

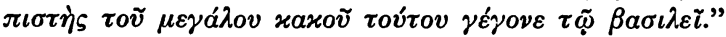

(9) G. Ostrogorsky, Les débuts de la querelle des images, in: Mélanges Charles Diehl I., 
Paris 1930 , p. 236 spricht von ihm als einer legendären Person im Gegensatz zu A. A. Vasiliev, The iconoclastic edict of the caliph Yazid II. a. d. 721, in: DOP. 9/10, 1956, 23-47, besonders p. 31, Anm. 17. Siehe ferner Beck, a. a. O., p. 33 und Anm. 4, J. Starr, An Iconodulic Legend and its Historical Basis, in: Speculum 8, 1933, 500-503, N. Jorga, Les origines de l'iconoclame, in: Bulletin Sect. Hist. Acad. Roumaine 11, 1924, 143-156., L. Bréhier, Beser, in: Dictionnaire d'histoire et de géographie ecclésiastiques, VIII., 1935, 1171-1172.

(10) Theophanes, Chronographia, a. m. 6218 (de Boor, 405, 3-5):

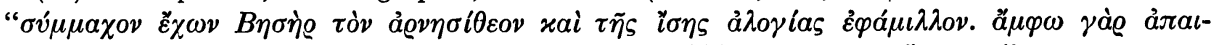

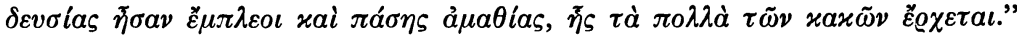

(11) Theophanes, Chronographia, a.m. 6233 (de Boor, 414, 26-28):

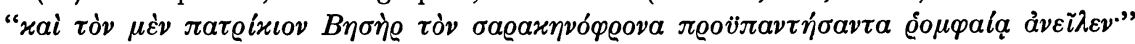

(12) vgl. A. Vasiliev, a. a. O., p. 28, Anm. 11 mit Literaturangabe.

(13) vgl. A. Vasiliev, a. a. O., p. 28, Anm. 12 mit Literaturangabe.

(14) Der dreijährige Unterschied kann ruhig dahingestellt bleiben, da der Unterschied für unsere Fragestellung kein wesentlicher ist.

(15) Theophanes, Chronographia, a.m. 6218 (de Boor, 405, 13-14):

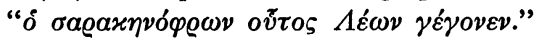

(16) Über die bisherige Diskussion über die Echtheitsfrage siehe am besten E. Caspar, Papst Gregor II. und der Bilderstreit, in: Zeitschrift für Kirchengeschichte 52, 1933, 29-89, insbesondere pp. 29-31 mit der Literaturangabe. Die Zeilenangabe des Briefs wird nach der Ausgabe von Caspar, a. a. O., pp. 72-84 zitiert.

(17) Caspar, a. a. O., p. 73:

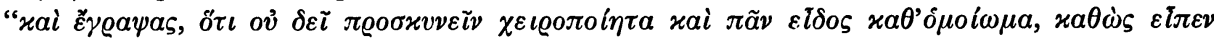

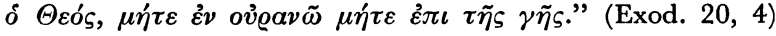

(18) Caspar, a. a. O., p. 77:

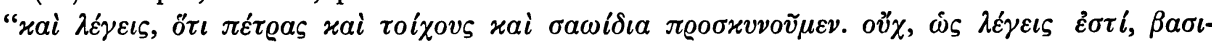
$\lambda \varepsilon \tilde{v}, x \tau \lambda . "$

(19) Caspar, a. a. O., p. 78;

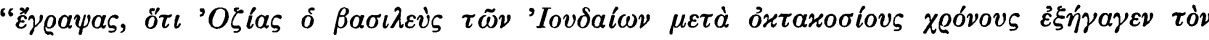

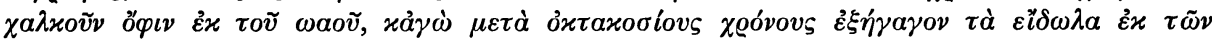

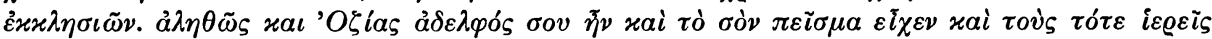

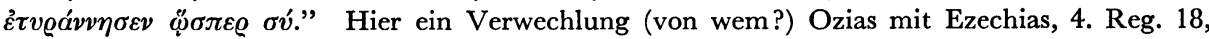
4. vgl. Caspar, a. a. O., p. 41, Anm. 32.

(20) Über die Verbreitung des Bilderkultes in Byzanz siehe J. Kollwitz, Bild III. (christlich), in: RAG. II. 318-341 mit Literaturangabe., V. Grumel, Images, in: DThC. 7, 766-844.

(21) vgl. dazu Kollwitz, a. a. O., p. 326, E. v. Dobschütz, Christusbilder I., I.eipzig 1899,

(22) vgl. dazu Theophanes, Chronographia, a. m. 6094 (de Boor 285, 4-17), Caspar, a. a. O., pp. 49-50 und Anm. 47 a bis Anm. 51.

(23) Oben wird die Ausgabe von G. Spulber, L'Eclogue des Isauriens. Texte, traduction, histoire, Cernautzi 1929 benutzt. Über die Ekloge siehe E. Pieler, Rechtsliteratur, in: Die hochsprachliche profane Literatur der Byzantiner II. hrsg. v. H. Hunger, München 1978, p. $438 \mathrm{ff}$.

(24) Spulber, a. a. O., p. 4:

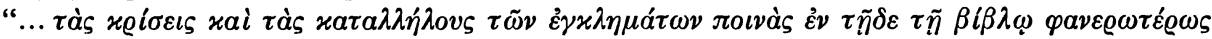

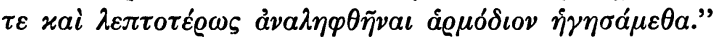

(25) Spulber, a. a. O., p. 6:

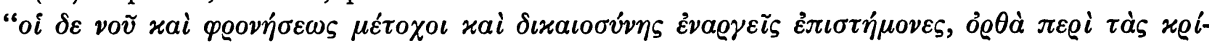

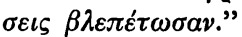

(26) Wie in der Anmerkung 2. angedeutet wird, sind bekanntlich bereits verschiedene Hypothesen und ihre Gegenansichten hinsichtlich der persönlichen Beweggründe dieses ikonoklastischen Kaisers vorgebracht worden: z. B. Betonung der Resonanz des kleinasiatischen Soldatentums 
mit dem Ikonoklasmus (F. Masai, La politique des Isauriens et le naissance de l'Europe, in: Byz. 23, 1963, 191-221), Hervorhebung der Feindschaft des Kaisers gegen den Großgrundbesitzertum der Kirche und des Klosters (N. Jorga, Les origines, =Anm. 9), Hinweis des Ikonoklasmus als eine seiner Reformtätigkeiten (Schenk, Kaiser Leo III., =Anm. 1), oder man lehnt diese Hypothesen ab und läßt die Lücke stehen (H. G. Beck, Die griechische Kirche, =Anm. 1). Unser Artikel will ein bescheidener Beitrag sein, um neben den bisherigen Hypothesen noch eine neue hinzustellen, die die dunkle Seite des Kaisers von einer bestimmten Seite erhellen und sie mehr ans Licht bringen will. 